Short communication

\title{
Order error in the calculation of continuous relative phase
}

\author{
Sina Mehdizadeh \& Paul S Glazier \\ National Sports Institute of Malaysia \\ Kuala Lumpur Sports City \\ Kuala Lumpur \\ Malaysia
}

Keywords: Coordination

Phase-plane portrait

Phase angle

Hilbert transform

Human movement kinematics

Sina Mehdizadeh is the corresponding author (sina.m@isn.gov.my)

Citation: $\quad$ Mehdizadeh, S. \& Glazier, P. S. (2018). Order error in the calculation of continuous relative phase. Journal of Biomechanics.

https://doi.org/10.1016/j.jbiomech.2018.03.032 


\begin{abstract}
The aims of this study were to demonstrate "order error" in the calculation of continuous relative phase (CRP) and to suggest two alternative methods-(i) constructing phase-plane portraits by plotting position over velocity; and (ii), the Hilbert transform - to rectify it. Order error is the change of CRP order between two degrees of freedom (e.g., body segments) when using the conventional method of constructing phase-plane portraits (i.e., velocity over position). Both sinusoidal and non-sinusoidal simulated signals as well as signals from human movement kinematics were used to investigate order error and the performance of the two alternative methods. Both methods have been shown to lead to correct results for simulated sinusoidal and non-sinusoidal signals. For human movement data, however, the Hilbert transform is superior for calculating CRP.
\end{abstract}




\section{Introduction}

Over the past few decades, an increasing number of studies in the biomechanics and motor control literatures have attempted to quantify the coordination, or coupling relationship, among the component parts, or degrees of freedom (DOF), of the human movement system. At the behavioural level of analysis, continuous relative phase (CRP) has been one of the most popular methods for quantifying intra- and inter-limb coordination. CRP represents the difference in the phase angles obtained from two DOF (e.g., thigh and shank angles during a gait cycle). To calculate CRP, phase-plane portraits are constructed for each DOF and the phase angle of one DOF is subtracted from the phase angle of the second DOF (Figure 1). However, while several studies have highlighted issues affecting the correct calculation and interpretation of CRP, such as range-normalisation (Hamill, van Emmerik, Heiderscheit, \& Li, 1999; Kurz \& Stergiou, 2002; Peters, Haddad, Heiderscheit, van Emmerik, \& Hamill, 2003), joint vs. segment angles (Lamb \& Stöckl, 2014), and discrete vs. cyclic movements (Lamb \& Stöckl, 2014), little attention has been paid to the appropriate construction of phase-plane portraits to achieve a correct order of CRP between segments.

To demonstrate this issue, consider the sinusoidal signal $x(t)$ in equation (1):

$$
x(t)=A \sin \left(\omega_{x} t+\theta_{x}\right)
$$

where $t, A, \omega_{x}$, and $\theta_{x}$ are time, magnitude, frequency, and phase shift, respectively.

The conventional method of calculating the phase angle is, first, to construct a phase-plane portrait by plotting the signal, $x$, on the abscissa axis, against its time derivative, $\dot{x}$, on the ordinate axis. Then, the phase angle is determined as the angle between the line connecting the origin to a point on the phase-plane trajectory and the right horizontal. Therefore, we have:

$$
\begin{gathered}
\phi_{x}(t)=\arctan \left(\frac{\dot{x}(t)}{x(t)}\right) \\
=\arctan \left(\frac{A \omega \cos \left(\omega_{x} t+\theta_{x}\right)}{A \sin \left(\omega_{x} t+\theta_{x}\right)}\right)
\end{gathered}
$$

where, $\phi_{x}$ is the phase angle of signal $x$, and $\dot{x}$ is the time derivate of $x$. After range-normalising $\omega$ (Peters et al., 2003), we have:

$$
\begin{aligned}
\phi_{x}(t) & =\arctan \left(\frac{\cos \left(\omega_{x} t+\theta_{x}\right)}{\sin \left(\omega_{x} t+\theta_{x}\right)}\right) \\
& =\arctan \left(\cot \left(\omega_{x} t+\theta_{x}\right)\right)
\end{aligned}
$$

It is evident from Equation ( $3 b$ ) that the inverse tangent of a cotangent function will not result in the associated angle. Indeed, since $\cot (\alpha)=\tan \left(\frac{\pi}{2}-\alpha\right)$, Equation (3b) reads: 

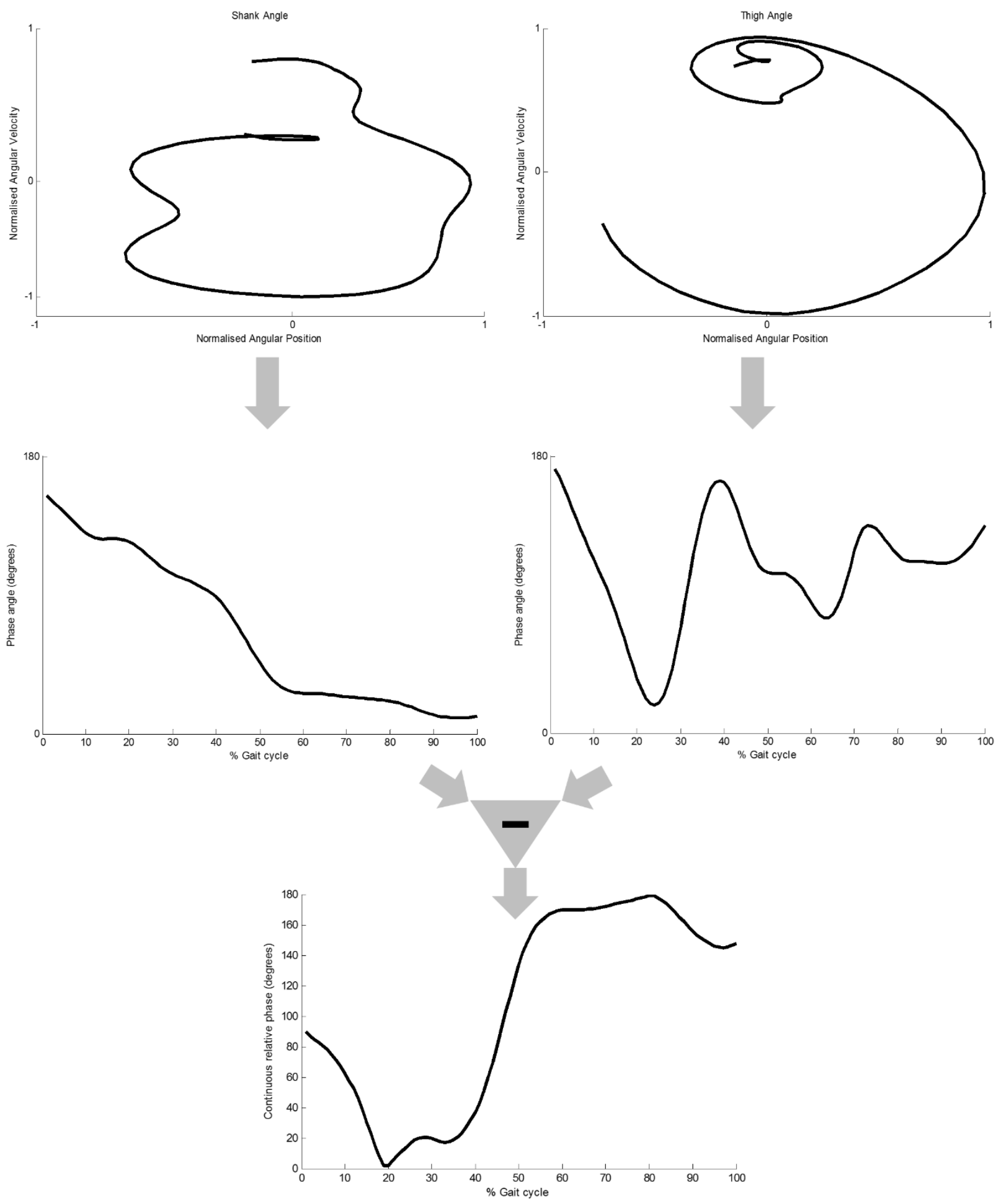

Figure 1. The conventional method of calculating continuous relative phase (CRP) using exemplar gait data: (top row) phase-plane portraits are constructed from normalized angular displacement and angular velocity each segment; (middle row) phase angles are then calculated from the phase-plane trajectories of each segment; (bottom row) finally, CRP is calculated by subtracting the phase angle of the distal segment (shank) from the phase angle of the proximal segment (thigh) at each instant of time. 


$$
\begin{aligned}
\phi_{x}(t) & =\arctan \left(\tan \left(\frac{\pi}{2}-\left(\omega_{x} t+\theta_{x}\right)\right)\right) \\
& =\frac{\pi}{2}-\left(\omega_{x} t+\theta_{x}\right)
\end{aligned}
$$

In other words, using the conventional method of constructing a phase-plane portrait and calculating the phase angle, there is a $90^{\circ}$ shift in the phase angle. Similarly, for the sinusoidal signal, $y(t)$, the phase angle is:

$$
\phi_{y}(t)=\frac{\pi}{2}-\left(\omega_{y} t+\theta_{y}\right)
$$

To calculate the CRP between signals $x(t)$ and $y(t)$ (i.e., $\Delta \phi_{x-y}$ ), the phase angle of signal $y(t)$ should be subtracted from the phase angle of signal $x(t)$. Therefore, we have:

$$
\begin{aligned}
\Delta \phi_{x-y} & =\phi_{x}(t)-\phi_{y}(t) \\
& =\frac{\pi}{2}-\left(\omega_{x} t+\theta_{x}\right)-\left(\frac{\pi}{2}-\left(\omega_{y} t+\theta_{y}\right)\right) \\
& =\left(\omega_{y} t+\theta_{y}\right)-\left(\omega_{x} t+\theta_{x}\right)
\end{aligned}
$$

In a special case such as human walking where $\omega_{x} \cong \omega_{y}=\omega$, equation (6c) reads:

$$
\begin{aligned}
\Delta \phi_{x-y} & =\left(\omega t+\theta_{y}\right)-\left(\omega t+\theta_{x}\right) \\
& =\theta_{y}-\theta_{x}=\Delta \phi_{y-x} \quad \perp
\end{aligned}
$$

where $\perp$ shows contradiction. In other words, with the conventional method, we get an "order error". That is, while the intention was to determine the CRP in $\mathrm{x}-\mathrm{y}$ order, the result is an $\mathrm{y}-\mathrm{x}$ order or $180^{\circ}$ phase shifted. While this artefact is not important when calculating the absolute value of CRP, it becomes significant when preserving the negative and positive signs as they influence the qualitative interpretation of coordination patterns among DOF. For example, if the phase angle of the distal segment is subtracted from the phase angle of the proximal segment, then positive CRP values indicate that the proximal segment leads the distal segment. However, according to Equations (6a-c), the conventional approach of calculating phase angle provokes misleading results by changing the order of subtraction.

Two solutions could be proposed to rectify the order error: first, constructing phase-plane portraits by plotting position ( $x$, ordinate) over velocity ( $\dot{x}$, abscissa) and second, using Hilbert transform. By plotting position versus velocity (the alternative method), Equation ( 2 a) becomes:

$$
\begin{aligned}
\phi_{x} & =\arctan \left(\frac{x(t)}{\dot{x}(t)}\right) \\
& =\arctan \left(\tan \left(\omega t+\theta_{x}\right)\right) \\
& =\omega t+\theta_{x}
\end{aligned}
$$

Therefore, the $90^{\circ}$ phase shift of the phase angle no longer exists and thus $\Delta \phi_{x-y}=\theta_{x}-\theta_{y}$. 
Calculation of the phase angle using the Hilbert transform introduced by Lamb and Stöckl (2014) (see also Rosenblum \& Kurths, 1998, and Varlet \& Richardson, 2011) is also an appropriate method to overcome order error. According to this method, the signal $x(t)$ is first transformed to an analytic signal $\zeta$ using Hilbert transform:

$$
\zeta=x(t)+i H(t)
$$

where the Hilbert transform $H(t)$ of $x(t)$ serves as the imaginary part of the analytic signal.

Then, the phase angle at each time point $\phi_{x}$ is calculated as:

$$
\phi_{x}=\arctan \left(\frac{H(t)}{x(t)}\right)
$$

Finally, the CRP between signals $x(t)$ and $y(t)$ (i.e. $\Delta \phi_{x-y}$ ), is calculated as $\Delta \phi_{x-y}=\phi_{x}-\phi_{y}$. This method has also been used to determine intra-limb coordination in human movement (Lamb \& Stöckl, 2014; Mehdizadeh, Arshi, \& Davids, 2015). The CRP calculated using the Hilbert transform is free of frequency artefacts (Lamb \& Stöckl, 2014).

The aim of this study was, therefore, to demonstrate order error and the virtues of the two alternative methods discussed. The feasibility of the two suggested methods are also indicated and discussed. Both sinusoidal and non-sinusoidal simulated signals as well as signals from human movement kinematics were used to investigate order error.

\section{Methods}

\subsection{Sinusoidal signals}

Two sinusoidal signals $x(t)=\sin (2 t), t \in[0,2 \pi]$ and an $18^{\circ}$ phase delayed signal $y(t)=$ $\sin \left(2 t-18^{\circ}\right)$ which have previously been used in coordination studies (Lamb \& Stöckl, 2014), were adopted in this study (Figure 2).

\subsection{Non-sinusoidal signals}

The two non-sinusoidal signals used in this study were (Lamb \& Stöckl, 2014; Peters et al., 2003):

$$
x(t)=\frac{\cos (t-0.25 \pi)}{\sqrt{1+0.41418^{2}-2 \times 0.41418 \sin (t-0.25 \pi)}}, t \in[0,2 \pi]
$$

and $y(t)$ as the $126^{\circ}$ phase shifted version of $x(t)$ (Figure 3). 


\subsection{Human movement kinematic data}

Sagittal plane shank and thigh angles of a young healthy male were calculated while walking at his preferred walking speed (Figure 4).

\subsection{Calculating CRP}

CRP was calculated using the three methods discussed in the introduction, i.e. by plotting velocity over position (conventional method; Hamill, et al., 1999), by plotting position over velocity (alternative method), and with the Hilbert method (Lamb \& Stöckl, 2014). In all cases, to calculate CRP, signal $y(t)$ (shank for kinematic signal) was subtracted from signal $x(t)$ (thigh for kinematic signal).

\section{Results}

\subsection{Sinusoidal signals}

Figure 2 shows the two sinusoidal signals, and CRP calculated using all three methods. As signal $x(t)$ is $18^{\circ}$ degree ahead of $y(t), \Delta \phi_{x-y}$ should be a straight line on $18^{\circ}$. However, calculating the phase angle using the conventional method resulted in a $180^{\circ}$ shift (i.e. $-18^{\circ}$ ). Using the alternative and also Hilbert methods, on the other hand, led to correct results.

\subsection{Non-sinusoidal signals}

Considering non-sinusoidal signals (Figure 3), since signal $y(t)$ is $126^{\circ}$ shifted with respect to $x(t), \Delta \phi_{x-y}$ should be oscillating around $126^{\circ}$. The results using the conventional method, however, led to CRP oscillating around $-126^{\circ}$ while using the alternative method as well as the Hilbert transform resulted in CRP oscillating around $126^{\circ}$.

\subsection{Human movement signals}

The thigh and shank signals are illustrated in Figure 4. It is evident that the thigh signal leads the shank signal. However, of the three methods, only the Hilbert transform calculated positive values for thigh-shank CRP. Moreover, the CRP patterns calculated using the traditional and alternative methods were different from each other and the Hilbert method.

\section{Discussion}

The aims of this study were to: (i) demonstrate order error in the calculation of CRP when using the conventional method of constructing phase-plane portraits (velocity over position); and (ii) to examine two solutions (i.e., the alternative construction of the phase-plane portrait by plotting position over velocity and the Hilbert transform) to rectify the order error. The feasibility of the suggested methods was explored using sinusoidal and non-sinusoidal signals as well as signals from human movement kinematics. 

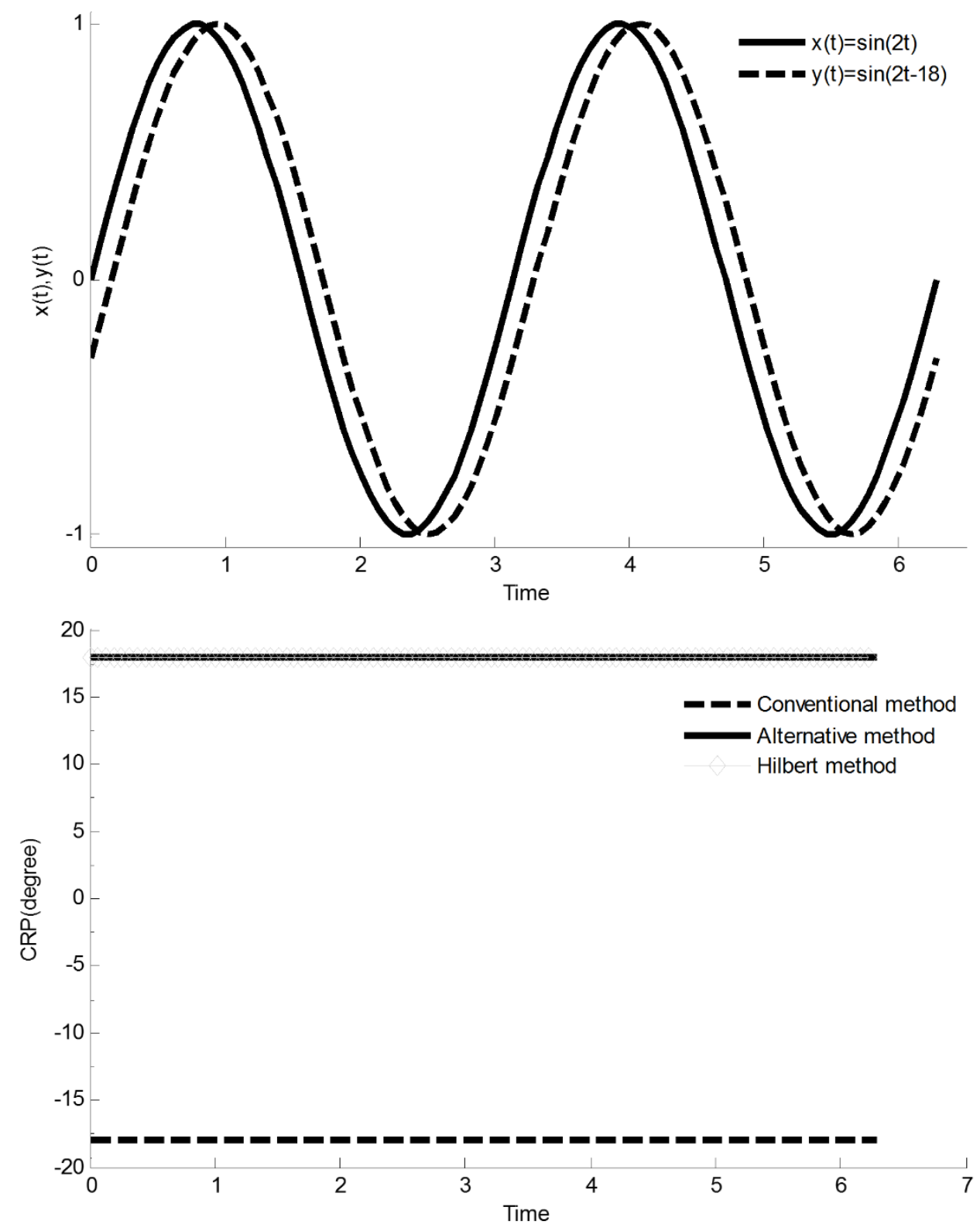

Figure 2. Two simulated sinusoidal signals where signal $x(t)$ is $18^{\circ}$ ahead of signal $y(t)$ (top), and the corresponding CRP calculated using conventional, alternative, and Hilbert methods (bottom). Note that the CRP lines for the Hilbert and alternative methods coincide. 

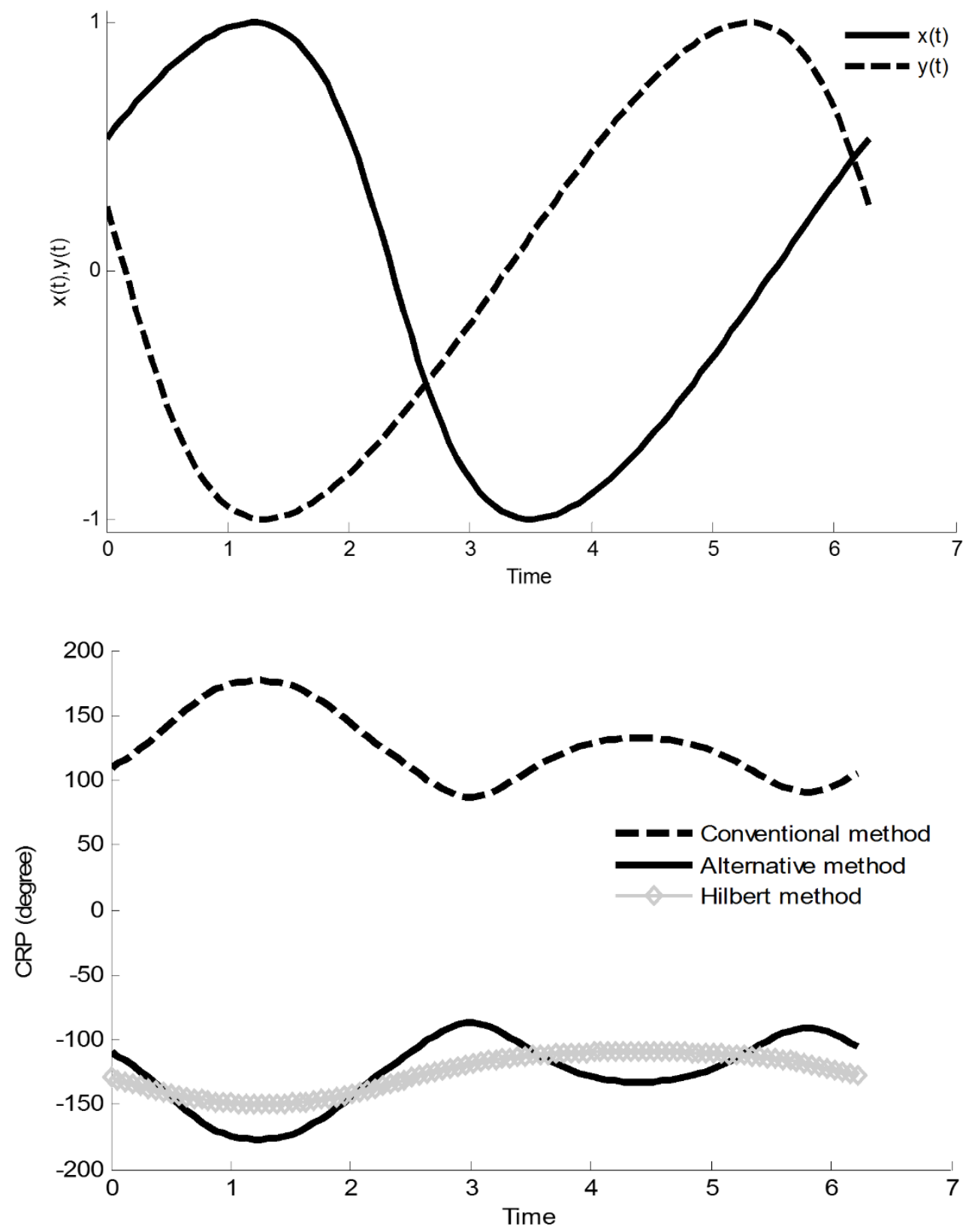

Figure 3. Two simulated non-sinusoidal signals where signal $y(t)$ is $126^{\circ}$ shifted with respect to of signal $x(t)$ (top), and the corresponding CRP calculated using conventional, alternative, and Hilbert methods (bottom). 

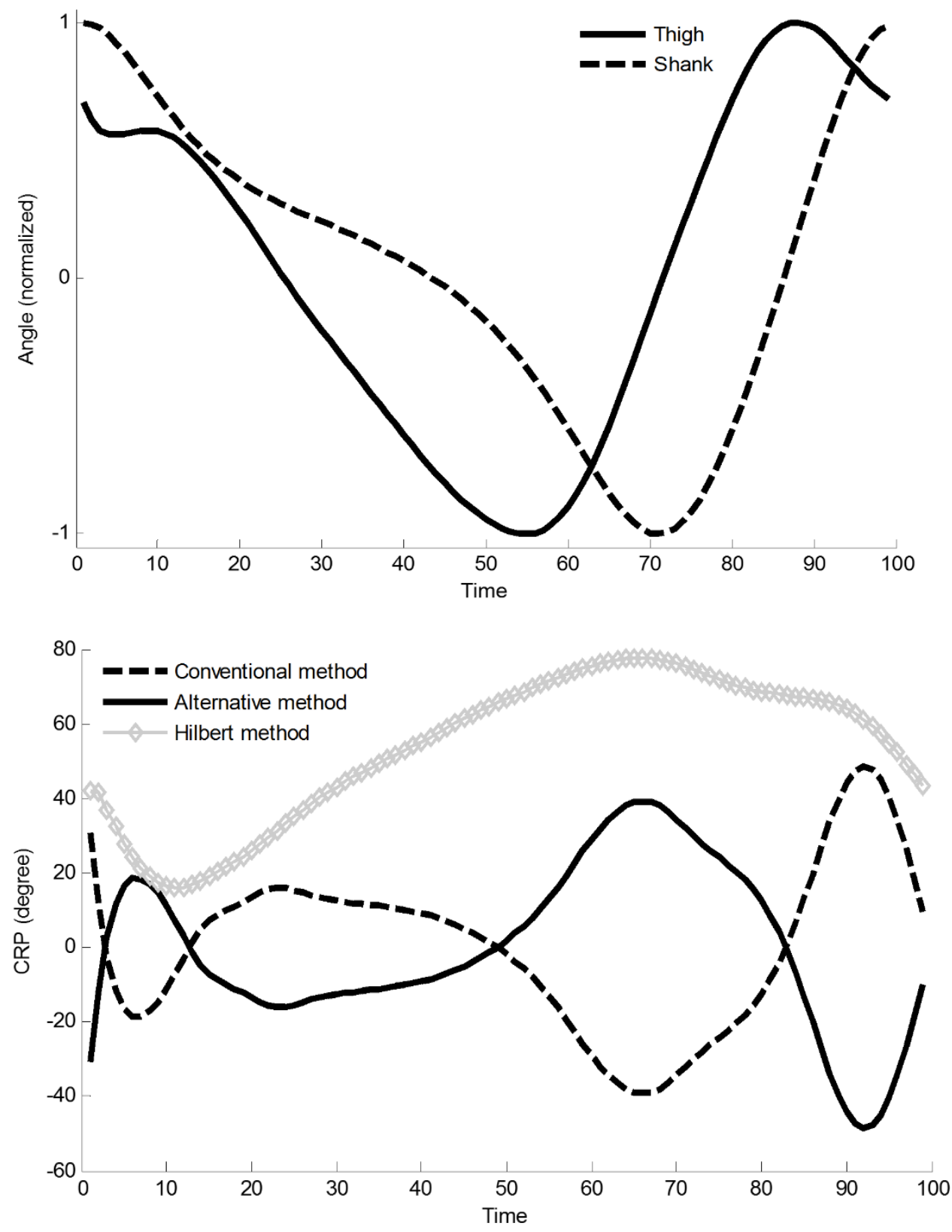

Figure 4. Normalised thigh and shank signals (top), and the corresponding CRP calculated using conventional, alternative, and Hilbert methods (bottom).

Our findings indicate that both the alternative and Hilbert transform methods are appropriate procedures for rectifying order error as it was analytically demonstrated in the Introduction. In our study, we used simulated sinusoidal and non-sinusoidal signals which have been utilised previously (Lamb \& Stöckl, 2014; Peters et al., 2003). However, the results of these studies are comparable to our findings using the conventional method of constructing phase-plane portraits 
showing the presence of order error in the previous literature (e.g., see Figure 4 of Peters et al., 2003, and Figures 1 and 3 of Lamb \& Stöckl, 2014, and compare to results of our study).

For the signals of human movement kinematics, our results (Figure 4) indicate that, although both the alternative method and the Hilbert transform are superior to the conventional method, the Hilbert transform yields better results. This is due to the fact that, in contrast to simulated signals which only contain one frequency in their frequency spectrum, signals of human movement are comprised of a range of different frequencies. Thus, range-normalising of segmental angular velocity using approaches suggested in previous studies (Hamill, Haddad, \& McDermott, 2000; Kurz \& Stergiou, 2002; Lamb \& Stöckl, 2014; Peters et al., 2003) will not resolve issues with frequency artefacts. Using the Hilbert transform, on the other hand, will account for this issue as it has previously been demonstrated by Lamb and Stöckl (2014) that the CRP calculated using Hilbert transform is free of frequency artefacts. Therefore, we suggest that when calculating CRP for human movement signals which exhibit significant non-sinusoidal behaviour, the Hilbert transform is a better choice. The only issue with the Hilbert transform is that the phase-plane portraits and phase angles cannot be shown visually as the Hilbert transform alters the signal to a complex space containing real and imaginary components which cannot be depicted on the real axes.

One point to mention here is that another suitable method of quantifying coordination could be cross-correlation. However, cross-correlation is based on the assumption that there is a linear relationship within segments or joints, which is not always the case. If the data are nonlinear, a transformation such as a $\log -\log$ transformation is required to linearize the data. However, if the coefficient of cross-correlation is still small after the transformation (implying that there is still no linear relationship between the segments of interest), cross-correlation is not suitable. Another disadvantage of the cross-correlation technique is that it provides only one measure per movement cycle (Wheat \& Glazier, 2006).

In summary, the implication of our findings is that, in addition to issues such as normalising and frequency artefacts investigated in previous studies (Hamill et al., 2000; Kurz \& Stergiou, 2002; Lamb \& Stöckl, 2014; Peters et al., 2003), consideration needs to be given to, and precautions taken to avoid, order error. This issue, however, is only important when the order of coordination of the moving segments (i.e., the sign of CRP value) is of concern. When the objective is to calculate the absolute value of CRP, the order error is of little consequence.

\section{Conclusion}

This study showed that the conventional method of constructing phase-plane portraits (i.e. velocity over position) leads to order error in calculating CRP. That is, this method will change the order of coordination from a proximal-distal order to a distal-proximal order or vice versa. Two alternative approaches are suggested to rectify order error: (i) constructing phase-plane portraits by plotting position over velocity; (ii), use the Hilbert transform. Both solutions have been shown to lead to correct results for simulated sinusoidal and non-sinusoidal signals. For human movement data, however, the Hilbert transform is superior for calculating CRP.

\section{Conflict of interest statement: None}




\section{Acknowledgement}

The authors would like to thank M.A. Sanjari from Iran University of Medical Sciences for his valuable comments on the equations of this study.

\section{References}

Hamill, J., Haddad, J. M., \& McDermott, W. J., 2000. Issues in quantifying variability from a dynamical systems perspective. Journal of Applied Biomechanics 16, 407-418. doi: 10.1123/jab.16.4.407

Hamill, J., van Emmerik, R. E. A., Heiderscheit, B. C., \& Li, L., 1999. A dynamical systems approach to lower extremity running injuries. Clinical Biomechanics 14(5), 297-308. doi: 10.1016/S0268-0033(98)90092-4

Kurz, M. J., \& Stergiou, N., 2002. Effect of normalization and phase angle calculations on continuous relative phase. Journal of Biomechanics 35(3), 369-374. doi: 10.1016/S00219290(01)00211-1

Lamb, P. F., \& Stöckl, M., 2014. On the use of continuous relative phase: Review of current approaches and outline for a new standard. Clinical Biomechanics 29(5), 484-493. doi: 10.1016/j.clinbiomech.2014.03.008

Mehdizadeh, S., Arshi, A. R., \& Davids, K., 2015. Quantifying coordination and coordination variability in backward versus forward running: Implications for control of motion. Gait \& Posture 42(2), 172-177. doi: 10.1016/j.gaitpost.2015.05.006

Peters, B. T., Haddad, J. M., Heiderscheit, B. C., Van Emmerik, R. E. A., \& Hamill, J., 2003. Limitations in the use and interpretation of continuous relative phase. Journal of Biomechanics 36(2), 271-274. doi: 10.1016/S0021-9290(02)00341-X

Rosenblum, M., \& Kurths, J., 1998. Analyzing synchronization phenomena from bivariate data by means of the Hilbert Transform. In: Kantz, H., Kurths, J., Mayer-Kress, G. (Eds.), Nonlinear Analysis of Physiological Data. Springer, Berlin, pp. 91-99.

Varlet, M., \& Richardson, M.J., 2011. Computation of continuous relative phase and modulation of frequency of human movement. Journal of Biomechanics 44, 1200-1204. doi: 10.1016/j.jbiomech.2011.02.001

Wheat, J.S. \& Glazier, P.S., 2006. Measuring coordination and variability in coordination. In: Davids, K., Bennett, S. \& Newell, K., (eds.) Movement system variability. Human Kinetics, 167-181. 